\title{
Design of a wireless intelligent device for Metal oxide arrestor on-line monitoring by using DSP \\ Xiaotong YAO ${ }^{1, a}$, Jirong WANG ${ }_{1, b}$,Yawen LI ${ }_{1, c}$
}

${ }^{1}$ Electronic and Information Engineering Institute, Lanzhou Jiao tong University

Lanzhou, 730070,Gansu, China

*Correspondence to Jirong WANG,Izjt12@163.com

National Natural Science Foundation of China [51567014]

\author{
Keywords: Metal Oxide Surge Arrester (MOA),On-line monitoring, DSP, Resistive \\ current.FFT,VC6.0.
}

\begin{abstract}
Monitoring of metal oxide surge arresters (MOA) due to aging, moisture and other components cause increased resistive current. Therefore, this paper designed an experiment-based DSP on-line monitoring device. The instrument can transmit the data into our computers or cell phonespassed by WIFI . In order to detect the operating state of MOA, we design a real-time monitoring interface by VC6.0 .This device uses resistive current as a detection targetand we can calculate the value by FFT, The main monitoring parameters are the fundamental and peak value of resistive current, third harmonic content of the leakage current, phase angle difference and power consumption. Through laboratory tests, the device can be used with a network arrester line monitoring, realization the data transmission of remote, reducing the risk of inspection personnel and the economic losses caused by power outages and improve the distribution network reliability.
\end{abstract}

\section{Introduction}

The MOA with non-linear characteristics like the large flow capacity, no series gaps, small size, light weight and excellent features gradually replaced the traditional SiC.Its main role is to absorb over-voltage,operating the energy impanct of over-voltage and prevent over-voltage substation to damage the electrical equipments. Under normal operating voltage, the current flowing through the arrester is few micro amps, when applied over-voltage the flow of current through the arrestor reached thousand of amps while surge arresters is in the conduction state ${ }^{[1]}$. The release of over-voltage energy, while limiting the amplitude, which badly violates the over-voltage power transmission equipment, after than we resumed the zinc oxide varistor in impedance state, so that the power system will back to normal operation. Due to continuous operating voltage arrester long-term effects, if the product has too high chargeability arrester beyond capacity, it will accelerate the aging of resistors, resistive current and subsequent increase in the power consumption that cause a crash, subsequent heat lightning breakdown or explosion. Internal moisture is caused by a lightning arrester which is important factor in accident.

In this paper, we design a wireless intelligent device for MOA on-line monitoring by using DSP, the device uses resistive leakage current component as a detection object. By the lab experiments, we observe the operation of the online monitoring system fullfil the requirements of the arrester.

\section{The equivalent circuit of MOA}

The equivalent circuit of MOA is shown in Fig . $1^{[2]}$. 


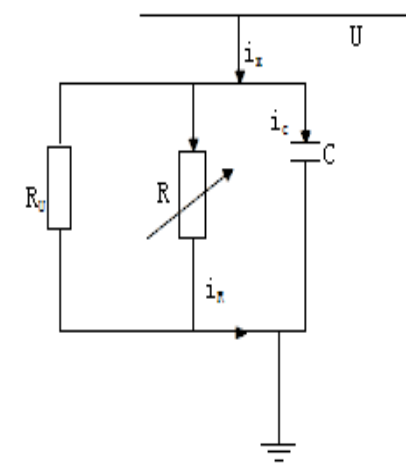

Fig .1 The equivalent circuit of MOA

In Fig .1, $\mathrm{R}$ is the equivalent nonlinear resistance; $\mathrm{C}$ is a constant equivalent capacitance; $i_{R}$ is resistive leakage current; $i_{c}$ is capacitive leakage currents; $i_{x}$ is the total leakage current; $U$ is the operating voltage of MOA.

\subsection{The model of the MOA}

In this paper,We chose the typical index model of the $\mathrm{MOA}^{[2]}$. The expression is $\mathrm{i}_{b}=p\left(u_{b} / u_{\text {ref }}\right)^{q}$.

$\mathrm{p}$ and $\mathrm{q}$ is the characteristic constant,Uref is the reference voltage of MOA. The graph for MOA resistive current average voltage characteristic as shown in Fig.2.

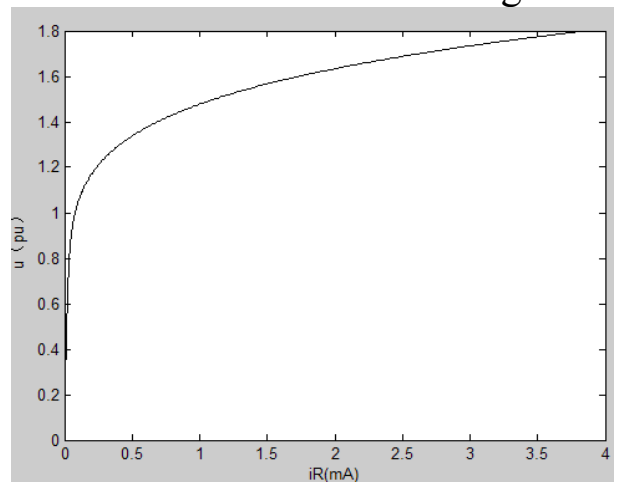

Fig.2 The graph for MOA resistive current average voltage characteristic

In Fig.2, The $\mathrm{u}(\mathrm{u}=\mathrm{U} / \mathrm{UN}$, the uint is $\mathrm{pu})$ is represents by vertical axis and $i_{R}$ (resistive leakage current) is represents by horizontal axis.

\section{The design of arrester on line monitoring device}

\subsection{The hardware of MOA on line monitoring device}

In this paper,we chose the resistive current as a detection target.We used some digital waveform analysis techniques like harmonic analysis and digital filtering software interference method that measure very accurate and stable results. It can also analyse the component of the fundamental and harmonic for 3 7 times,overcome interference and correctly measure the resistive current of $\mathrm{MOA}^{[3]}$. In this way you can judge the MOA's initial operating conditions. The final control system is shown in Fig.3. 


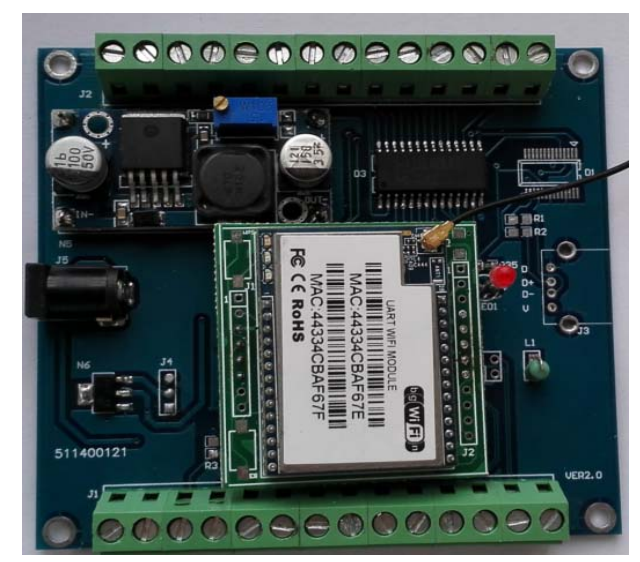

Fig .3 The hardware of MOA on line monitoring device

In order to meet the requirements of the precision of the system. The TMS320F28335 of DSP and the ADS1256 has be choosed. The ADS1256 are extremely low-noise, 24-bit analog-to-digital (A/D) converters ${ }^{[4,5]}$. They provide complete high-resolution measurement solutions for the most demanding applications. Monitoring the final results and using the transation data with WIFI into the laptop, cellphone and IPAD.

\subsection{The sampling subroutine of AD}

\section{(1)The algorithm of DFT}

The extraction algorithms of resistive current, the value of fundamental and third harmonic can be calculated by FFT ${ }^{[6,7]}$. Discrete Fourier transform is a signal from the time domain into the frequency domain and it is one of the indispensable tools about transformation in the form of digital signal processing. If the sample for the whole period, the DFT is calculated as follows:

$$
X(K)=A_{K}+j B_{K}=\frac{2}{N}\left(\sum_{n=0}^{N-1}(x) \cos \frac{2 \pi n k}{N}-j \sum_{n=0}^{N-1} x(n) \sin \frac{2 \pi n k}{N}\right) \cdot
$$

Where, $N$ is the number of samples, $X(K)$ is the harmonic of $k-t h, x(n)$ is the sampling value of n-th .

In this paper, a periodic sampling is 128 , the sampling frequency is $6400 \mathrm{~Hz}$, the four cycles can be continuous sampled, the toatal sampling points is $512(\mathrm{~N}=512)$, the resolution of frequency is $\Delta f=12.5 \mathrm{~Hz}$, when the frequency is $50 \mathrm{~Hz}, \mathrm{k}$ is 4 ,and the frequency is $150 \mathrm{~Hz}, \mathrm{k}$ is 12 . The expression is

$$
\begin{aligned}
& X(4)=A_{4}+j B_{4}=\frac{1}{256}\left(\sum_{n=0}^{511} x(n) \cos \frac{\pi n}{64}-j \sum_{n=0}^{511} x(n) \sin \frac{\pi n}{64}\right) \cdot \\
& X(12)=A_{12}+j B_{12}=\frac{1}{256}\left(\sum_{n=0}^{511} x(n) \cos \frac{3 \pi n}{64}-j \sum_{n=0}^{511} x(n) \sin \frac{3 \pi n}{64}\right) \cdot
\end{aligned}
$$

Since the cosine function is a periodic function,we can calculate the value of the first cycle by equation 3 and 4 .

\section{(2)A / D sampling}

In this design, the main purpose of us is sample the voltage and the resistance current of MOA,each sample has two signals, operating voltage and leakage current of MOA, requirement sample at the same time.Each cycle has 125 points, the four cycles is sampled. The A / Dsampling flow chart is shown in Fig.4. 


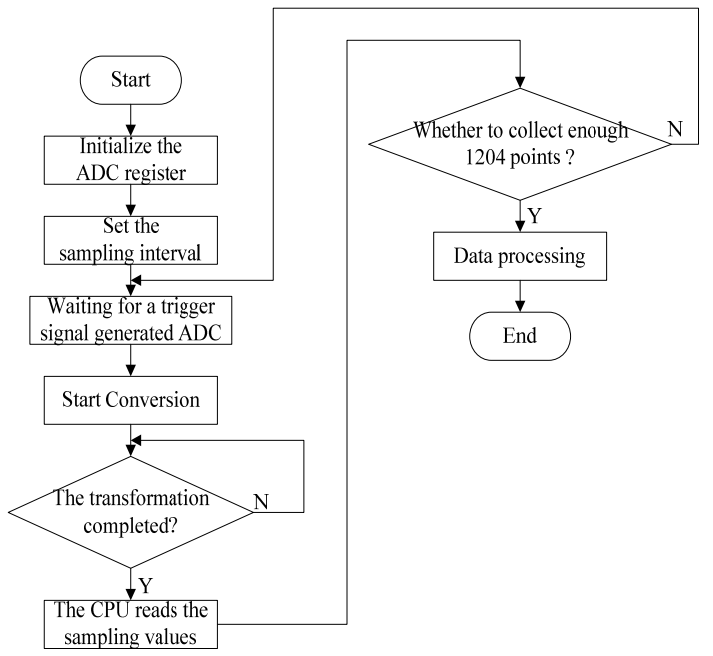

Fig.4 A / Dsampling flow chart

The $U_{2}=\sin (100 \pi t)+0.2 \sin (300 \pi t)$ and random noise signal can be simulation of the grid system voltage signal, as shown in Fig .5. When the current contains the harmonics than it can be described in Fig .6a and without the harmonics that it can be described in Fig .6b.

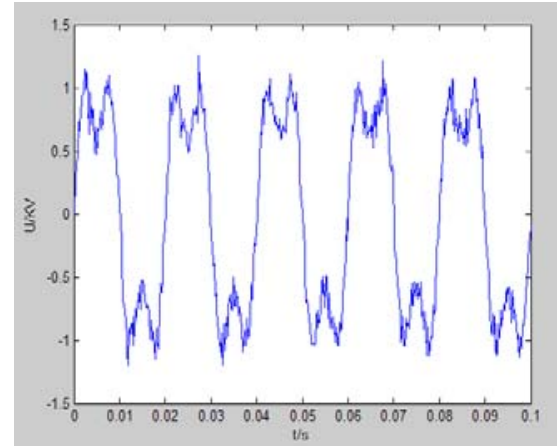

Fig .5 The analog of grid voltage signal

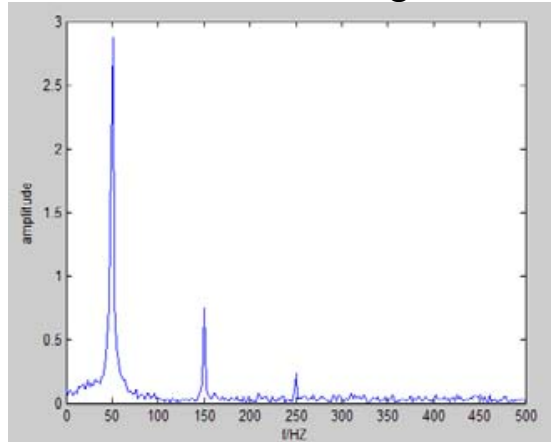

Fig .6a Resistive current with the $3^{\text {rd }}$ and $5^{\text {rd }}$ harmonic frequency characteristics

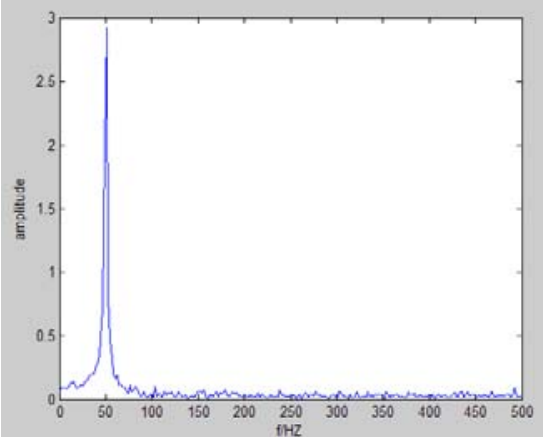

Fig .6b Resistive current without the $3^{\text {rd }}$ and $5^{\text {rd }}$ harmonic frequency characteristics

Fig. 6 The analog of current amplitude-frequency characteristic

From the above, we can conclude when the nonlinear resistors valve contains the three and five harmonic of leakage current in the grid, the three and five harmonic amplitudes of leakage current is larger than the wihout the three and five harmonic of grid, they change with nonlinear resistance valves' change. Therefore, We can determine the operating status of MOA through the size of the third and fifth harmonic of resistance. In the actual operation we can plot the waveform of the three and five harmonic amplitudes of the grid and harmonic amplitude of resistive leakage about the MOA.Through the comprehensive analysis, we can conclude the state of MOA.

\section{The results of MOA-line monitoring}

The MOA of 10KV (HY5WS-17/50 type) has be used for our laboratory test ,This device can test the effectiveness and motion effects.Through testing transformer can adjust the voltage on the 
arrester; the testing transformer variable ratio is 500:1. In this design, we use VC6.0 wrote a real-time monitoring interface. This monitoring interface is shown in Fig.7 .

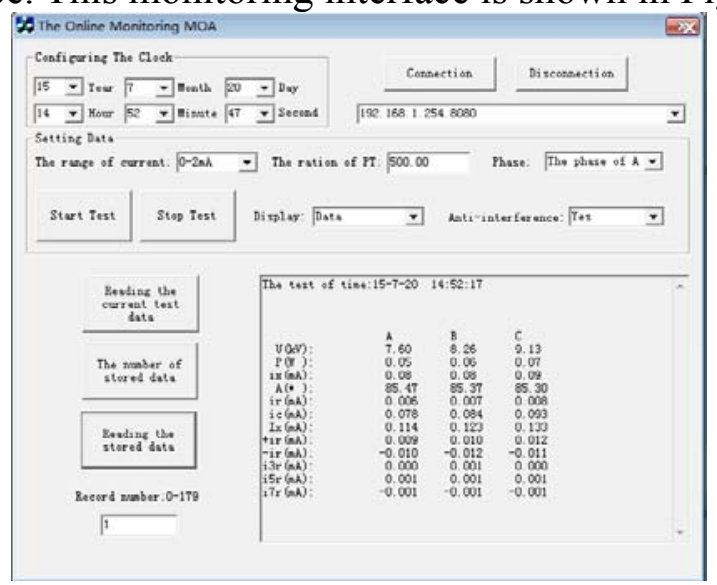

Fig.7 The monitoring interface of MOA

In the Fig.11, $\mathrm{U}$ is the the test voltage, $\mathrm{P}$ is the power consumption of MOA,ix is the total current, $\mathrm{A}$ is the phase angle difference, ir is the resistive current,ic is the capacitive current,Ix is the peak total current,$+i$ ir the positive peak resistive current,-ir is the negative peak resistive current, is the $3^{\text {rd }}$ of resistive leakage current,i5r and $i 7 r$ is similar the $i 3 r$.

Depend on the above tests, we obtained some results that are as follow:

(1)In the system voltage, the full current is $0.078 \mathrm{~mA}$ and the resistive current component is $0.006 \mathrm{~mA}$. We can see that the resistive current is only $13 \%$ of full current.Some researchers explained that the resistance current is about $10 \% \sim 20 \%$ of the total current under the normal conditions, if the test values will be in this range then it shows that MOA is working well. If resistance current is about $25 \% \sim 40 \%$ of all current then that will increase the testing frequency and pay close attention to transformation, do data analysis and judgment.If resistance current is more than $40 \%$ of all current, it is consider out of operation.

(2)Through above data we observed that the value of the resistive current for current is very small and the total content of capacitive current is very large.

(3)By testing we observed that the performance of MOA is good, so in this case the $i_{3 R}, i_{5 R}$ and $i_{7 R}$ are consider 0 .

\section{5 . Conclusion}

The $10 \mathrm{KVof}$ MOA is used in the laboratory for on-line monitoring testing and the monitoring interface written by VC6.0 . The data is transfered into the computer by WIFI and it can be detected during MOA aging,moisture and resistive current component that can be used to detect hidden arrester insulation in time. The resistive current component is consider as detection target for auto-monitoring device, using a digital waveform analysis technique, such as harmonic analysis and digital filtering software anti-interference methods to make the measurement results accurate and stable. It can accurate analysis the fundamental wave and harmonic content for $3 \sim 7$ times,overcome the interphase interference effects and correctly measure the resistance of arrester current.Based on these values of longitudinal, securityand transverse comparison, we concluded that the MOA is in aging or may be affected with damp. This can determine whether or not to stop the working of MOA and reduce the damage for electric power system.

\section{References}

[1]. Zhang Zhenhong,Zang Dianhong.Study on the On-line Monitoring Method of Metal Oxide Surge Arrester[J].High Voltage Apparatus.2009(05).

[2]. Xiong Pengcheng, Zhou Wenjun, Su Xueyuan, Yi Xiao, Li Xiaolu, Yang Xia.Design of On-line Monitoring Device for MOA Used in $10 \mathrm{kV}$ Distribution Network[J].High Voltage 
Engineering.2007(08)

[3]. Liu Huijia, Li Ning.Research on Detection Method of MOA Resistence Leakage Current Based on Harmonic Analysis[J].J of China Three Gorges Univ1(Natural Sciences).2003, 23 (2) : 144-147.

[4]. $\mathrm{Xu}$ Zhiniu, Zhao Lijuan,Ding Ao, Lu Fangcheng.Calculation of MOA resistive current[J].Electric Power Automation Equipment.2010(12).

[5]. Zhu Hanxin, Raghuveer M R. Influence of representation model and voltage harmonics on metal oxidesurge arrester diagnostics[J]. IEEE Trans. on Power Dilivery, 2001, 16(4): 599-603.

[6]. Zhu Hanxin, Raghuveer M R. Influence of harmonics in system voltage on metal oxide surge arrester diagnostics[C]. 1999 Conferences on Electrical Insulation and Dielectric Phenomena, Atlanta, USA,1999, 2: 542-545.

[7]. Xiaolei Yu, Yongrong Sun, Jianye Liu, and Jianfeng Miao. Design and realization of synchronization circuit for GPS software receiver based on DSP .Journal of Systems Engineering and Electronics, Vol. 21, No. 1, February 2010, pp.20-26. 